\title{
Human milk oligosaccharide consumption by probiotic and human-associated bifidobacteria and lactobacilli
}

\author{
Taksawan Thongaram, ${ }^{* 1}$ Jennifer L. Hoeflinger, ${ }^{*}$ JoMay Chow, $†$ and Michael J. Miller ${ }^{* 2}$ \\ *Department of Food Science and Human Nutrition, University of Illinois, Urbana 61801 \\ †Abbott Nutrition, Columbus, $\mathrm{OH} 43219$
}

\begin{abstract}
Human milk contains high concentrations of nondigestible complex oligosaccharides (human milk oligosaccharides; HMO) that reach the colon and are subsequently fermented by the infant gut microbiota. Using a high-throughput, low-volume growth determination, we evaluated the ability of 12 lactobacilli and 12 bifidobacteria strains, including several commercial probiotics, to ferment HMO and their constituent monomers. Of the 24 strains tested, only Bifidobacterium longum ssp. infantis ATCC 15697 and Bifidobacterium infantis M-63 were able to ferment 3 '-sialyllactose, 6 '-sialyllactose, 2 '-fucosyllactose, and 3'-fucosyllactose. Bifidobacterium infantis M-63 degraded almost $90 \%$ of the 2 '-fucosyllactose but left most of the fucose in the supernatant, as detected by HPLC. Among bifidobacteria, only the $B$. infantis strains and Bifidobacterium breve ATCC 15700 were able to ferment lacto- $N$-neotetraose (LNnT). Among lactobacilli, Lactobacillus acidophilus NCFM was found to be the most efficient at utilizing LNnT. The extracellular $\beta$-galactosidase (lacL, LBA1467) of L. acidophilus NCFM cleaves the terminal galactose of LNnT for growth, leaving lacto- $N$-triose II in the media as detected by HPLC. Inactivation of lacL abolishes growth of L. acidophilus NCFM on LNnT. These results contribute to our knowledge of HMO-microbe interactions and demonstrate the potential for synbiotic combinations of pre- and probiotics.
\end{abstract}

Key words: human milk oligosaccharide, Bifidobacterium, Lactobacillus acidophilus NCFM, probiotic

\section{INTRODUCTION}

Human milk contains essential nutrients required for infant growth and development. Human milk oligosaccharides (HMO), the third most abundant component

\footnotetext{
Received February 17, 2017.

Accepted June 14, 2017.

${ }^{1}$ Current address: Department of Microbiology, Faculty of Science, Silpakorn University, Nakhonpathom 73000, Thailand.

${ }^{2}$ Corresponding author: mille216@illinois.edu
}

of human milk (approximately 5-23 g/L; Kunz et al., 2000; Zivkovic et al., 2011), consist of more than 200 complex linear and branched polymers of glucose (Glc), galactose (Gal), $\mathrm{N}$-acetylglucosamine (GlcNAc), fucose (Fuc), and $N$-acetylneuraminic acid (sialic acid, SA), with lactose (Lac) at the reducing end (Stahl et al., 1994; Ninonuevo et al., 2006). Preclinical data suggest that HMO confer multiple physiological benefits, including immunomodulation of the host (He et al., 2014), improved cognition (Vázquez et al., 2015), modulation of intestinal motility and perfusion (Bienenstock et al., 2013; Good et al., 2016), microbial production of short-chain fatty acids (Vester Boler et al., 2013) and other metabolites (Chow et al., 2014), and prevention of pathogen attachment (Kunz et al., 2000; Newburg et al., 2005). Moreover, HMO promote the growth of mucus-adapted and HMO-adapted microbes, such as bacteroides and bifidobacteria (Marcobal et al., 2010; Asakuma et al., 2011).

In addition to HMO, human milk contains bifidobacteria and lactobacilli (Fernández et al., 2013) that readily colonize the infant gastrointestinal tract (GIT; Sekirov et al., 2010). Formula-fed infants lack exposure to these potentially health-promoting oligosaccharides and microbes. Therefore, delivery of HMO and probiotic bifidobacteria and lactobacilli in infant formula or milk substitutes remains a priority. In vitro HMO utilization experiments revealed strain-dependent HMO fermentation among Bifidobacterium and Lactobacillus species. Specifically, Bifidobacteria longum ssp. infantis consumed fucosylated, sialylated, and Type I and II HMO (Ward et al., 2006; LoCascio et al., 2009; Asakuma et al., 2011; Yu et al., 2013; Garrido et al., 2015). Bifidobacterium bifidum and Bifidobacterium breve readily consume Type I and II HMO, whereas utilization of fucosylated and sialylated HMO is strain dependent (LoCascio et al., 2009; Asakuma et al., 2011; Ruiz-Moyano et al., 2013). On the other hand, Lactobacillus delbrueckii ssp. lactis moderately consumed fucosylated and sialylated HMO (Yu et al., 2013), and Lactobacillus casei BL23 utilized Type I HMO (Bidart et al., 2015). Lactobacillus casei, Lactobacillus acidophilus, and Lactobacillus plantarum possess enzymes 
Table 1. Microorganisms used in this study



${ }^{1} \mathrm{NA}=$ source of origin not publicly available.

${ }^{2}$ Isolated from commercially available ProTectis drops (BioGaia, Stockholm, Sweden).

capable of hydrolyzing HMO, but growth is not always supported (Rodríguez-Díaz et al., 2011; Schwab and Gänzle, 2011). For example, purified $\alpha$-L-fucosidases from L. casei BL23 liberate Fuc from 2'-fucosyllactose (2'-FL) in vitro (Rodríguez-Díaz et al., 2011), but it is unclear whether this occurs with whole cells. Additionally, L. casei BL23 coupled fermentation of fucosyl- $\alpha-$ 1,3-GlcNAc with excretion of Fuc, suggesting that the active fucosidases act intracellularly (Rodríguez-Díaz et al., 2012).

Given that human milk, containing HMO, bifidobacteria, and lactobacilli, is ingested by infants, we determined the fermentation parameters for various probiotic Bifidobacterium and Lactobacillus strains on purified HMO and HMO constituent monomers. Furthermore, we investigated the degradation pathway of lacto- $N$-neotetraose (LNnT) by L. acidophilus NCFM. Results from this study add to our current understanding of HMO-microbe interactions and could potentially lead to the development of truly synergistic, synbiotic combinations of pre- and probiotics.

\section{MATERIALS AND METHODS}

\section{Bacterial Strains and Growth Conditions}

Commercial probiotic and human-isolated bifidobacteria $(\mathrm{n}=12)$ and lactobacilli $(\mathrm{n}=12)$ strains (Table
1) were purchased from the American Type Culture Collection (ATCC), isolated from a commercial probiotic product, or supplied from the culture collection of Abbott Nutrition (Columbus, OH). Bacterial strains were cultured in de Man, Rogosa and Sharpe broth (Difco, Franklin Lakes, NJ) and incubated anaerobically $\left(90 \% \mathrm{~N}_{2}, 5 \% \mathrm{CO}_{2}\right.$, and $\left.5 \% \mathrm{H}_{2}\right)$ at $37^{\circ} \mathrm{C}$ for $24 \mathrm{~h}$. For bifidobacteria, all growth medium was supplemented with $0.05 \%$ (wt/vol) L-cysteine.

\section{Carbohydrate Utilization Assays}

Stationary phase cultures were twice subcultured in semidefined de Man, Rogosa and Sharpe (sMRS) broth (Barrangou et al., 2003) containing 1\% (wt/vol) Glc. Cells were washed twice with PBS, harvested by centrifugation at $3,220 \times g$ for $10 \mathrm{~min}$, and resuspended in $10 \mathrm{~mL}$ of carbohydrate-free sMRS. Cells were inoculated $(1 \%, \mathrm{vol} / \mathrm{vol})$ into sMRS containing $1 \%$ (wt/vol) of Glc, Gal, Lac, Fuc, GlcNAc, SA, 3'-sialyllactose (3'SL), 6'-sialyllactose (6'-SL), 2'-FL, 3'-fucosyllactose (3'-FL), LNnT, or no carbohydrate. All HMO were purchased from V-Labs Inc. (Covington, LA) except LNnT, which was provided by Abbott Nutrition. A honeycomb plate was prepared with $250 \mu \mathrm{L}$ of bacterial inocula covered with $50 \mu \mathrm{L}$ of mineral oil, held for $1 \mathrm{~h}$, and incubated anaerobically at $37^{\circ} \mathrm{C}$ for up to $120 \mathrm{~h}$. The change in optical density at $600 \mathrm{~nm}\left(\Delta \mathbf{O D}_{600}\right)$ was 
measured every 30 min using a Bioscreen $\mathrm{C}$ machine (Growth Curves USA, Piscataway, NJ) following maximum agitation for $30 \mathrm{~s}$. A minimum of 3 independent replications were completed for each carbohydrate. To confirm increases in OD correlated with bacterial growth, select bacterial and carbohydrate combinations were serially diluted and enumerated by surface plating on Reinforced Clostridial Medium (Difco).

\section{HMO Consumption, Postfermentation Analyses, and $\beta$-Galactosidase Activity}

Stationary-phase HMO-consuming bifidobacteria and lactobacilli cultures were twice subcultured in sMRS containing 1\% (wt/vol) Glc, washed in PBS, and inoculated $(1 \%, \mathrm{vol} / \mathrm{vol})$ into $3 \mathrm{~mL}$ of sMRS containing $1 \%$ (wt/vol) 2'-FL, 3'-SL, LNnT, or no carbohydrate. Cultures were incubated anaerobically at $37^{\circ} \mathrm{C}$ for 48 (2'-FL), 72 (3'-SL), or $96 \mathrm{~h}(\mathrm{LNnT})$, and $\Delta \mathrm{OD}_{600}$ was obtained with a spectrophotometer. Uninoculated medium was included as a control.

Following growth, cultures were centrifuged at 12,000 $\times g$ for $5 \mathrm{~min}$, and the cell-free supernatants were filter sterilized $(0.22 \mu \mathrm{m})$ and stored at $-20^{\circ} \mathrm{C}$. Three independent replicates of cell-free supernatants were analyzed by HPLC and thin-layer chromatography (TLC). For HPLC, endpoint samples were run on a 1260 Infinity HPLC (Agilent Technologies, Santa Clara, CA) equipped with a refractive index detector using a Rezex ROA-Organic Acid $\mathrm{H}^{+}$(8\%) column (Phenomenex, Torrance, CA). The column was eluted with $0.005 \mathrm{~N}$ $\mathrm{H}_{2} \mathrm{SO}_{4}$ at a flow rate of $0.6 \mathrm{~mL} / \mathrm{min}$ at $5^{\circ} \mathrm{C}$, and Fuc, SA, 2'-FL, 3'-SL, LNnT, and lacto- $N$-triose II (LNTri; Carbosynth US, San Diego, CA) were used as detection standards. For TLC, cell-free supernatants were obtained from $250-\mu \mathrm{L}$ culture aliquots removed at 24 , 48, 72, and $96 \mathrm{~h}$. Silica gel TLC plates were spotted with $2 \mu \mathrm{L}$ of cell-free supernatants; developed with a 2:1:1 ( $\mathrm{vol} / \mathrm{vol} / \mathrm{vol})$ mixture of 1-butanol, glacial acetic acid, and water; treated with an aerosolized mixture of diphenylamine, aniline, and phosphoric acid (85\%) in ethanol; and visualized by heat at $120^{\circ} \mathrm{C}$ for $5 \mathrm{~min}$ (Kocourek et al., 1966). Glucose, GlcNAc, Gal, Lac, LNnT, and LNTri were used as detection standards.

Extracellular $\beta$-galactosidase activity during growth on LNnT was measured at 24, 28, 72, and $96 \mathrm{~h}$ by combining $5 \mu \mathrm{L}$ of cell-free supernatant, $80 \mu \mathrm{L}$ of McIlvaine buffer (Ghose and Bisaria, 1987), and $15 \mu \mathrm{L}$ of $0.05 \mathrm{M}$ o-nitrophenyl- $\beta$-galactoside. Enzymatic reactions were incubated at $37^{\circ} \mathrm{C}$ for $10 \mathrm{~min}$ and quenched by $1 \mathrm{M} \mathrm{Na}_{2} \mathrm{CO}_{3}$, and endpoint optical density at 405 $\mathrm{nm}$ was measured with a plate reader. One unit of $\beta$-galactosidase activity was defined as the amount of enzyme needed to liberate $1 \mu \mathrm{mol}$ of $o$-nitrophenol/ $\min$.

\section{$\beta$-Galactosidase Gene Inactivation}

The $\beta$-galactosidase (lacL) gene (LBA1467) of Lactobacillus acidophilus NCFM (LA_NCFM) was inactivated by pORI-based homologous recombination as previously described (Luchansky et al., 1991; Russell and Klaenhammer, 2001). Published primer sequences, bgalF and bgalR, were modified by a $5^{\prime}$ addition of $B a m H I$ and $X b a \mathrm{I}$ restriction enzyme sequences. Inactivation of $\triangle l a c L$ in LA_NCFM was confirmed by junction fragment PCR with ori+ (5'-ATAATGAACTGTGCTGATTAC-3') and lacL- (5'-GGATCGTAGACTAAGAGCGC-3'; expected size of $1,385 \mathrm{bp}$ ) and ori- (5'-TTCAATCGCCAACGAATC-3') and lacL+ (5'-CACAATCCCAGTTCCTAGTG-3'; expected size of $1,315 \mathrm{bp}$ ). All primers were purchased from Integrated DNA Technologies (Coralville, IA). Utilization of Glc, Gal, Lac, and LNnT in LA_NCFM $\Delta l a c L$ was compared with the wild-type LA_NCFM as described previously.

\section{Statistical Analyses}

The differences in sugar concentrations and $\beta$-galactosidase activity detected in the cell-free supernatant were determined with SAS (version 9.4; SAS Institute) using the generalized linear model. When statistical significance in sugar concentrations was observed $(P<0.05)$, Tukey's honest significant difference post hoc mean separation was run to control for unequal sample sizes. When statistical significance in $\beta$-galactosidase activity was observed $(P<0.05)$, Dunnett's test was run to compare the $\beta$-galactosidase activity during growth $(24,48,72$, and $96 \mathrm{~h})$ with the initial $\beta$-galactosidase activity $(0 \mathrm{~h})$. All data satisfied the assumptions of normality and homogeneity of variance.

\section{RESULTS AND DISCUSSION}

\section{Few Strains Could Grow on Intact HMO or HMO Constituent Monomers}

When we assessed several probiotic and humanisolated strains of bifidobacteria for their ability to utilize defined carbohydrates as a sole carbon source, we observed differential consumption of intact HMO and HMO constituent monomers (Figure 1). Three clusters of Bifidobacterium spp. were revealed according to their differential ability to utilize the tested carbohydrates, 
and, notably, none of the tested bifidobacteria strains were able to grow on Fuc or SA. Cluster 1 was characterized by weaker fermentation of Glc and Lac by Bifidobacterium animalis ssp. lactis Bb-12, B. animalis ssp. lactis Bf-6, B. bifidum ATCC 29521, B. animalis ssp. lactis DSM 10140, B. longum BB536, B. animalis ssp. animalis ATCC 25527, and $B$. breve. Of note, this cluster had very limited growth when Gal was given as a sole carbohydrate. The observed fermentation pattern of B. bifidum ATCC 29521 (JCM 1255) in this study is consistent with others results (LoCascio et al., 2009; Asakuma et al., 2011; Garrido et al., 2015); however, this strain is not representative of the B. bifidum species due to mutations in key HMO utilization genes (Asakuma et al., 2011). Stronger fermentation of Glc, Gal, and Lac by Bifidobacterium adolescentis ATCC 15703 and B. breve ATCC 15700 (BB_15700) was characteristic of cluster 2; BB_15700 was able to weakly ferment LNnT and uniquely fermented GlcNAc. The observed preference for growth on LNnT and GlcNAc by BB_15700 in this study is consistent with previous studies (Ward et al., 2007; LoCascio et al., 2009; RuizMoyano et al., 2013). The third cluster, B. longum ssp. infantis M-63 (BI_M63) and B. longum ssp. infantis S12 ATCC 15697 (BI_15697), exhibited very strong growth on Glc, Gal, and Lac. Although this cluster was able to moderately ferment 2'-FL, 3'-FL, 3'-SL, 6'-SL, and LNnT, virtually no utilization of GlcNAc, Fuc, or SA was observed. Others have also reported minimal growth of BI_15697 in the presence of GlcNAc, Fuc (Ward et al., 2007; Asakuma et al., 2011), and SA (Ward et al., 2007). Overwhelmingly, our results support the conclusion that $B$. longum ssp. infantis strains are uniquely efficient HMO utilizers compared with most other commensal GIT bifidobacteria (Ward et al., 2006, 2007; LoCascio et al., 2009; Asakuma et al., 2011; Yu et al., 2013; Garrido et al., 2015). Initially, the divergence of $B$. longum subspecies, B. longum ssp. infantis and ssp. longum, was believed to be attributable to the lack of HMO utilization gene clusters in $B$. longum ssp. longum (Sela et al., 2008; LoCascio et al., 2010). However, several novel $\alpha$-fusosidases have been identified in B. longum ssp. longum and B. longum ssp. suis capable of utilizing fucosylated HMO (Bunesova et al., 2016; Garrido et al., 2016).

In contrast, the carbohydrate utilization pattern within the tested lactobacilli did not fall into distinct clusters (Figure 1B). Most lactobacilli strains grew in the presence of Glc, Gal, Lac, and GlcNAc, whereas utilization of Fuc, SA, and each HMO was limited. Specifically, Lactobacillus rhamnosus GG ATCC 53103 (LR_53103) and L. rhamnosus HN001 DR20 utilized Fuc, and L. plantarum LP-66 utilized SA. Of note, LNnT was the sole HMO that supported the growth of lactobacilli. Specifically, L. plantarum LP-66 and Lactobacillus reuteri DSM 17938 grew minimally in the presence of LNnT, whereas $L$. acidophilus La-5 (LA_La5) and LA_NCFM grew moderately on this carbohydrate.

Although HMO utilization by bifidobacteria has been highly studied, information regarding the utilization of HMO and their constituent monomers by lactobacilli remains limited. Similar to our study, Schwab and Gänzle (2011) observed minimal growth on Fuc and strong growth on GlcNAc by 4 different Lactobacillus species. Moreover, Yu et al. (2013) reported minimal growth on $2^{\prime}$-FL, $3^{\prime}-\mathrm{FL}$, and $6^{\prime}$-SL by $L$. delbrueckii ssp. lactis ATCC 7830 but no growth on fucosylated or sialylated HMO by LR_53103. Likewise, we did not observe the growth on HMO by LR_53103. Two studies have demonstrated enzymatic hydrolysis of $2^{\prime}$-FL, 3 '-FL, 3'-SL, 6'-SL, and lacto- $N$-tetraose by several Lactobacillus species, but neither study included HMO growth data (Rodríguez-Díaz et al., 2011; Schwab and Gänzle, 2011). A follow-up study suggests that the fucosidase activity by L. casei BL23 is intracellular (Rodríguez-Díaz et al., 2012). However, it is unclear whether the enzymatic hydrolysis reported by Schwab and Gänzle (2011) occurred within or outside the lactobacilli cells. Nevertheless, we can use their results to guide the interpretation of our data. Because 2 -FL, $3^{\prime}$-FL, 3'-SL, and 6'-SL contain either a single Fuc or SA linked to an Lac molecule, enzymatic hydrolysis by an extracellular fucosidase or sialidase would generate extracellular Lac with monomeric Fuc or SA. If we observed hydrolysis of 2'-FL, 3'-FL, 3'-SL, and 6'-SL by extracellular fucosidases or sialidases, we should have also observed growth on these HMO due to the liberated Lac. However, we did not observe growth of any lactobacilli on 2'-FL, 3'-FL, 3'-SL, and 6'-SL, whereas growth on Lac was observed in most lactobacilli (10/12 strains).

\section{Incomplete Consumption of HMO by Bifidobacteria and Lactobacilli}

Previously, we demonstrated that growth of bifidobacteria and lactobacilli correlated with increased acid production (Yang et al., 2017); however, total acid concentration does not establish which carbohydrate monomers are utilized. Because BI_M63 consumed 2'-FL and 3 '-SL but not the component monosaccharides, Fuc and SA (Figure 1A), we hypothesized that these monomers would accumulate in the BI_M63 cell-free supernatant during growth on 2 '-FL and 3 '-SL, respectively. Following growth on $2^{\prime}-\mathrm{FL}$ by BI_M63 $\left(\Delta \mathrm{OD}_{600}=1.267\right)$, we detected significantly less $2^{\prime}$-FL $(1.65 \pm 0.55 \mathrm{~g} / \mathrm{L} ; P<$ $0.0001)$ remaining in the cell-free supernatant $(\sim 89 \%$ 
A
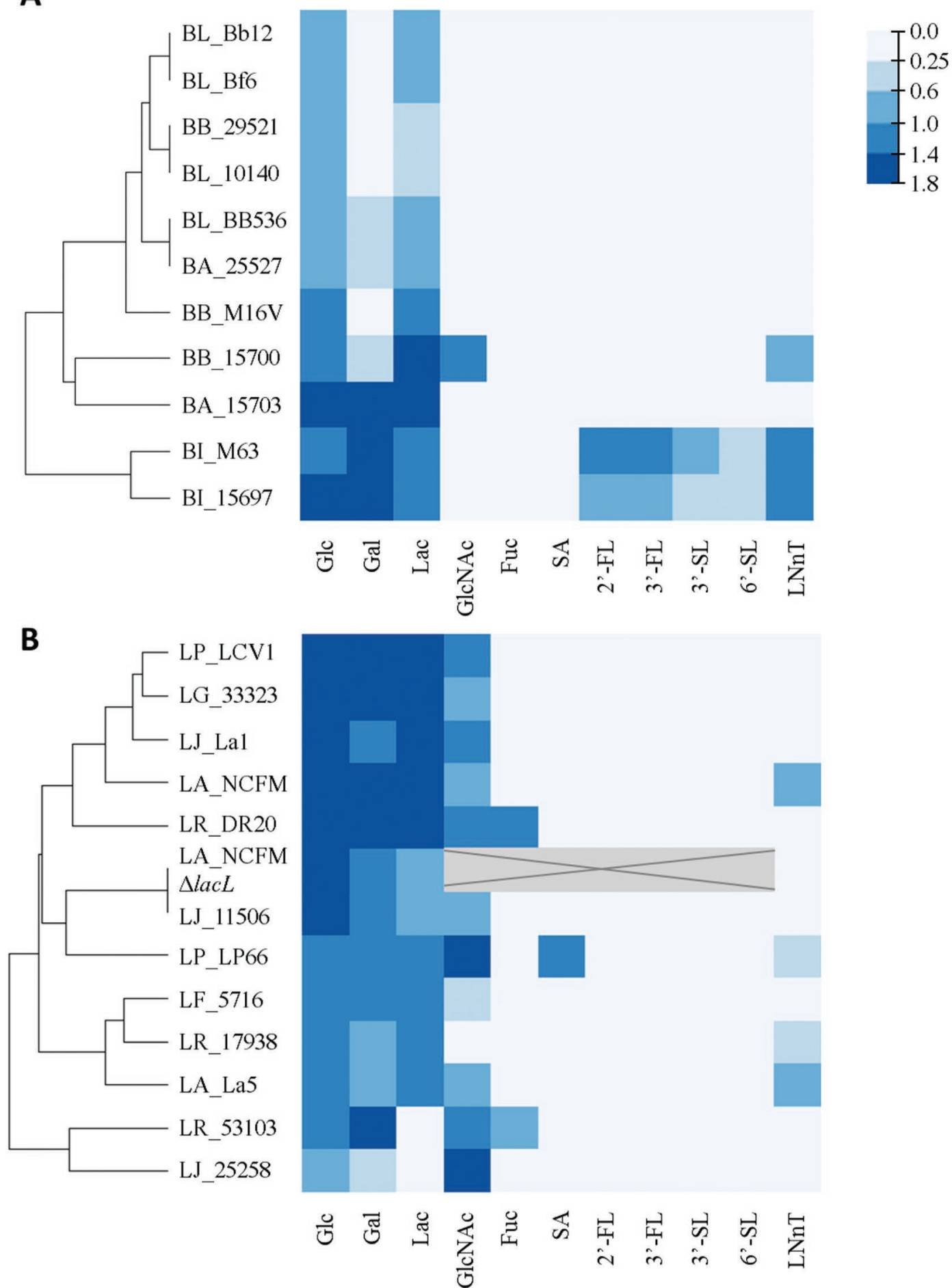

Figure 1. Maximum bacterial growth (change in optical density at $600 \mathrm{~nm}$ ) in the presence of $1 \%$ glucose (Glc), galactose (Gal), lactose (Lac), $N$-acetylglucosamine (GlcNAc), fucose (Fuc), $N$-acetylneuraminic acid (SA), 2'-fucosyllactose (2'-FL), 3'-fucosyllactose (3'-FL), $3^{\prime}$-sialyllactose (3'-SL), 6'-sialyllactose (6'-SL), and lacto- $N$-neotetraose (LNnT) in semidefined de Man, Rogosa and Sharpe (sMRS) broth (+0.05\% cysteine for bifidobacteria) for (A) bifidobacteria and (B) lactobacilli. Refer to Table 1 for strain abbreviations. All strains were able to grow in sMRS supplemented with $1 \%$ Glc, whereas, growth was minimal (change in optical density at $600 \mathrm{~nm} \leq 0.25$ ) in the absence of a carbohydrate (data not shown). Blank correction by subtraction of control wells without added cells or carbohydrates. Dendrogram shows relatedness by fermentation pattern. Grayed X box denotes carbohydrates not tested. Data are reported as the mean of 3 or more independent replicates. Color version available online. 
consumption; Figure 2A). Although little Fuc was detected in the uninoculated medium, Fuc was detected in the BI_M63 cell-free supernatant $(1.30 \pm 1.46 \mathrm{~g} / \mathrm{L}$; Figure 2A); however, it was not significantly different from the control $(P=0.1989)$. Of note, this accounted for approximately $55 \%$ of the Fuc initially added as part of 2'-FL. Conversely, BI_M63 moderately fermented 3 '-SL $\left(\Delta \mathrm{OD}_{600}=0.736\right)$, and significantly less $3^{\prime}$-SL $(2.99 \pm 0.87 \mathrm{~g} / \mathrm{L} ; P<0.0001)$ remained in the cell-free supernatant ( $\sim 68 \%$ consumption; Figure $2 \mathrm{~B})$. Although $0.97 \pm 0.42 \mathrm{~g} / \mathrm{L}$ of SA was detected in the BI_M63 cell-free supernatants, it was not significantly different than the SA detected in the carbohydrate-free sMRS and uninoculated controls $(0.83 \pm 0.06$ and 0.76 $\pm 0.52 \mathrm{~g} / \mathrm{L}$, respectively; $P=0.8070)$. Therefore, we conclude that BI_M63 does not accumulate SA in the fermentate. Consistent with the growth data (Figure 1A), BI_15697 and BI_M63 were efficient LNnT utilizers, with little accumulation of LNTri in the cell-free supernatant (Figure 2C). We detected $1.11 \pm 0.15$ $\mathrm{g} / \mathrm{L}(\sim 87 \%$ consumption) and $0.95 \pm 0.14 \mathrm{~g} / \mathrm{L}(\sim 89 \%$ consumption) of LNnT in the cell-free supernatants of BI_15697 and BI_M63, respectively, which was significantly lower than the control $(P<0.0001)$. Previously, 2 B. bifidum strains accumulated excess Fuc during growth on pooled and purified fucosylated HMO (Ward et al., 2007; Asakuma et al., 2011) by an unknown mechanism. Additionally, BI_15697 accumulated HMO constituent monomers, including Glc, Gal, GlcNAc, and Fuc, briefly during exponential growth followed by a disappearance during the stationary phase (Asakuma et al., 2011). The authors speculated that monosaccharides were excreted to minimize intracellular osmotic pressure caused by the rapid uptake of HMO. Wholegenome analysis of BI_15697 revealed the presence of a Fuc permease (Blon_2307) along with the absence of a Fuc catabolic pathway (Sela et al., 2008). It is plausible that the accumulation of Fuc by BI_M63, combined with its inability to utilize Fuc as a sole carbon source, is similar to BI_15697. Additionally, BI_M63 may have excreted Fuc to reduce osmotic pressure, accounting for the $55 \%$ of Fuc we detected in the cell-free supernatant.

In contrast to BI_15697 and BI_M63, LA_La5 and LA_NCFM utilized all LNnT component monosaccharides (Glc, Gal, and GlcNAc), whereas growth on LNnT was moderate (Figure 1B). Therefore, we hypothesized that LNnT is not fully consumed by LA_La5 and LA_NCFM. We detected significantly less $\operatorname{LNnT}(3.91 \pm 0.27$ and $0.61 \pm 0.06 \mathrm{~g} / \mathrm{L} ; P<$ $0.0001)$ by HPLC following growth of LA_La5 ( $53 \%$ consumption) and LA_NCFM ( $\sim 93 \%$ consumption), respectively (Figure 2C). Lacto- $N$-neotetraose is an oligosaccharide that comprises 4 monomers: Gal- $\beta-1,4-$ GlcNAc- $\beta-1,3$-gal- $\beta$-1,4-Glc. We detected significantly more LNTri (GlcNAc- $\beta-1,3$-gal- $\beta 1,4 \mathrm{Glc}$ ) in the cellfree supernatant of LA_NCFM $(2.35 \pm 0.06 \mathrm{~g} / \mathrm{L} ; P$ $<0.0001)$ compared with the LNTri $(<1.0 \mathrm{~g} / \mathrm{L})$ detected by HPLC in the cell-free supernatant of LA_La5 (Figure 2C). Consequently, we conducted a follow-up time-course experiment to observe the accumulation of LNTri during growth on LNnT (Figure 2D). Following fermentation of LNnT for $24 \mathrm{~h}$, LNTri was observed in the cell-free fermentate of LA_NCFM, and additional LNTri accumulated over the duration of the experiment. Similarly, LA_La5 slowly ( $>48$ h) accumulated minimal quantities of LNTri as detected by TLC (Figure 2D), which is consistent with the amount of LNTri $(0.18 \mathrm{~g} / \mathrm{L})$ detected in the cell-free supernatant by HPLC (Figure 2C). Miwa et al. (2010) demonstrated sequential degradation of LNnT from its nonreducing terminus by extracellular exoglycosidases from $B$. bifidum. An extracellular $\beta$-galactosidase (BbgIII) from B. bifidum JCM 1254 hydrolyzed LNnT into Gal and LNTri. Subsequent catabolism of LNTri occurred by 2 membrane-bound $\beta$ - $N$-acetylhexosaminidases (BbhI and BbhII; Miwa et al., 2010). Therefore, we hypothesized that hydrolysis of LNnT and the accumulation of LNTri by LA_NCFM occurred due to the action of an extracellular $\beta$-galactosidase.

\section{Lactobacillus acidophilus NCFM Requires an Extracellular $\beta$-Galactosidase to Utilize LNnT}

Our observations of LNTri accumulation led us to hypothesize that LA_NCFM possesses an extracellular glycosidase that liberates the terminal Gal for growth and discards LNTri. We periodically measured the extracellular $\beta$-galactosidase activity in the LA_La5 and LA_NCFM cell-free supernatants during growth on LNnT (Figure 3). The extracellular $\beta$-galactosidase activity in the LA_La5 cell-free supernatants remained low $(<0.02$ units $/ \mathrm{mL})$ through $72 \mathrm{~h}$ (Figure $3 \mathrm{~B})$. An increase in extracellular $\beta$-galactosidase activity corresponded with the exponential phase of LA_La5 growth. Likewise, the maximum extracellular $\beta$-galactosidase activity of $0.037 \pm 0.01$ units $/ \mathrm{mL}$ occurred at $96 \mathrm{~h}$ and was significantly more activity than at $0 \mathrm{~h}(P=0.0021)$. Due to the delay in extracellular $\beta$-galactosidase activity in LA_La5, we speculate that release of intracellular $\beta$-galactosidase during growth may account for the minimal extracellular $\beta$-galactosidase detected at 72 to $96 \mathrm{~h}$. In contrast, we detected a significant increase in extracellular $\beta$-galactosidase activity in the cell-free supernatants of LA_NCFM at all time points compared with the activity detected at $0 \mathrm{~h}(P<0.0001$; Figure $3 \mathrm{~A}$ ). This increased activity corresponded with exponential growth. The extracellular $\beta$-galactosidase activity of LA_NCFM increased to a maximum of 
A



B

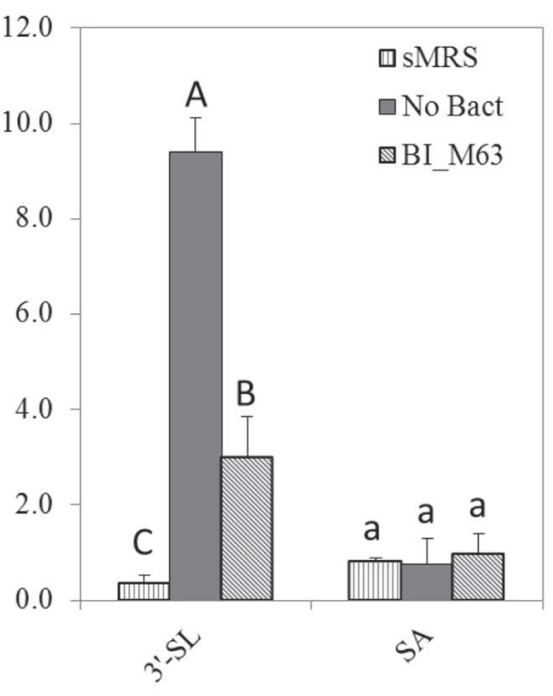

C

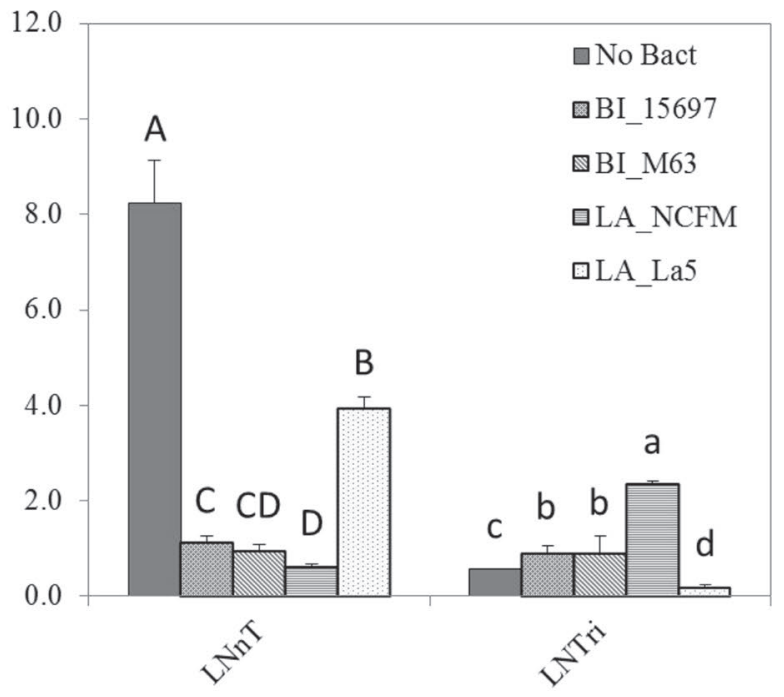

D

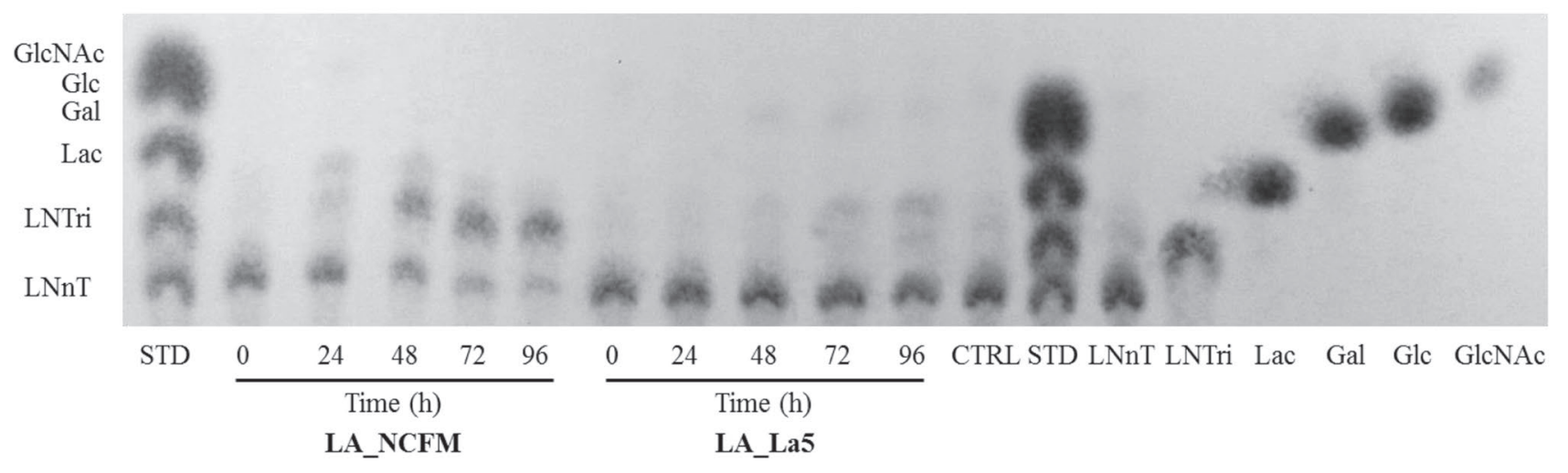

Figure 2. Presence of human milk oligosaccharides and their degradation products present in the cell-free supernatant of Bifidobacterium longum ssp. infantis M63 (BI_M63), B. longum ssp. infantis S12 ATCC 15697 (BI_15697), Lactobacillus acidophilus La-5 (LA_La5), and L. acidophilus NCFM (LA_NCFM). Concentration (g/L), by HPLC, of (A) 2'-fucosyllactose (2'-FL) and fucose (Fuc), (B) 3'-sialyllactose (3'-SL) and $N$-acetylneuraminic acid (SA), and (C) lacto- $N$-neotetraose (LNnT) and lacto- $N$-triose II (LNTri). No Bact $=$ cell-free supernatant from uninoculated well; sMRS $=$ growth media with no added carbohydrates. Data are reported as the mean \pm standard error of 3 independent replicates. (D) Time-course analysis, by thin-layer chromatography (TLC), of LNnT consumption by LA_NCFM and LA_La5. Glucose (Glc), galactose (Gal), lactose (Lac), $N$-acetylglucosamine (GlcNAc), lacto- $N$-triose II, and LNnT were included as standards. CTRL $=$ control (cellfree supernatant from uninoculated well); STD = ladder of standard carbohydrates. The TLC plate image was representative of 3 independent experiments. Values with no common letters $(\mathrm{a}-\mathrm{d} ; \mathrm{A}-\mathrm{D})$ are significantly different $(P<0.05)$.

$0.07 \pm 0.003$ units $/ \mathrm{mL}$ at $48 \mathrm{~h}$ and remained elevated through $96 \mathrm{~h}$ (>0.05 units $/ \mathrm{mL})$. Previously, we demonstrated that the extracellular $\beta$-galactosidase LacL (LBA1467) of LA_NCFM efficiently cleaved Gal from the nonreducing end of galactooligosaccharides, leading to accumulation of Gal in the cell-free supernatant of LA_NCFM (Thongaram et al., 2017). Furthermore, we hypothesized that LacL was responsible for liberating Gal from LNnT to support the growth of LA_NCFM. Following inactivation, LA_NCFM $\Delta l a c L$ was unable to utilize LNnT as a sole carbon source (Figure 1B). We conclude that LacL from LA_NCFM is responsible for hydrolysis of LNnT to Gal and LNTri. Although LacL
(LBA1467) was predicted to be localized intracellularly (Andersen et al., 2011), we believe that LacL from LA_NCFM cleaves LNnT extracellularly. It is possible that LacL is released during LA_NCFM cell turnover. However, we observed extracellular $\beta$-galactosidase activity that correlated with growth of LA_NCFM on LNnT (Figure 3A) rather than increased enzymatic activity at the end of growth, like LA_La5 (Figure 3B).

\section{CONCLUSIONS}

This work demonstrates differential utilization of intact HMO and HMO constituent monomers among the 

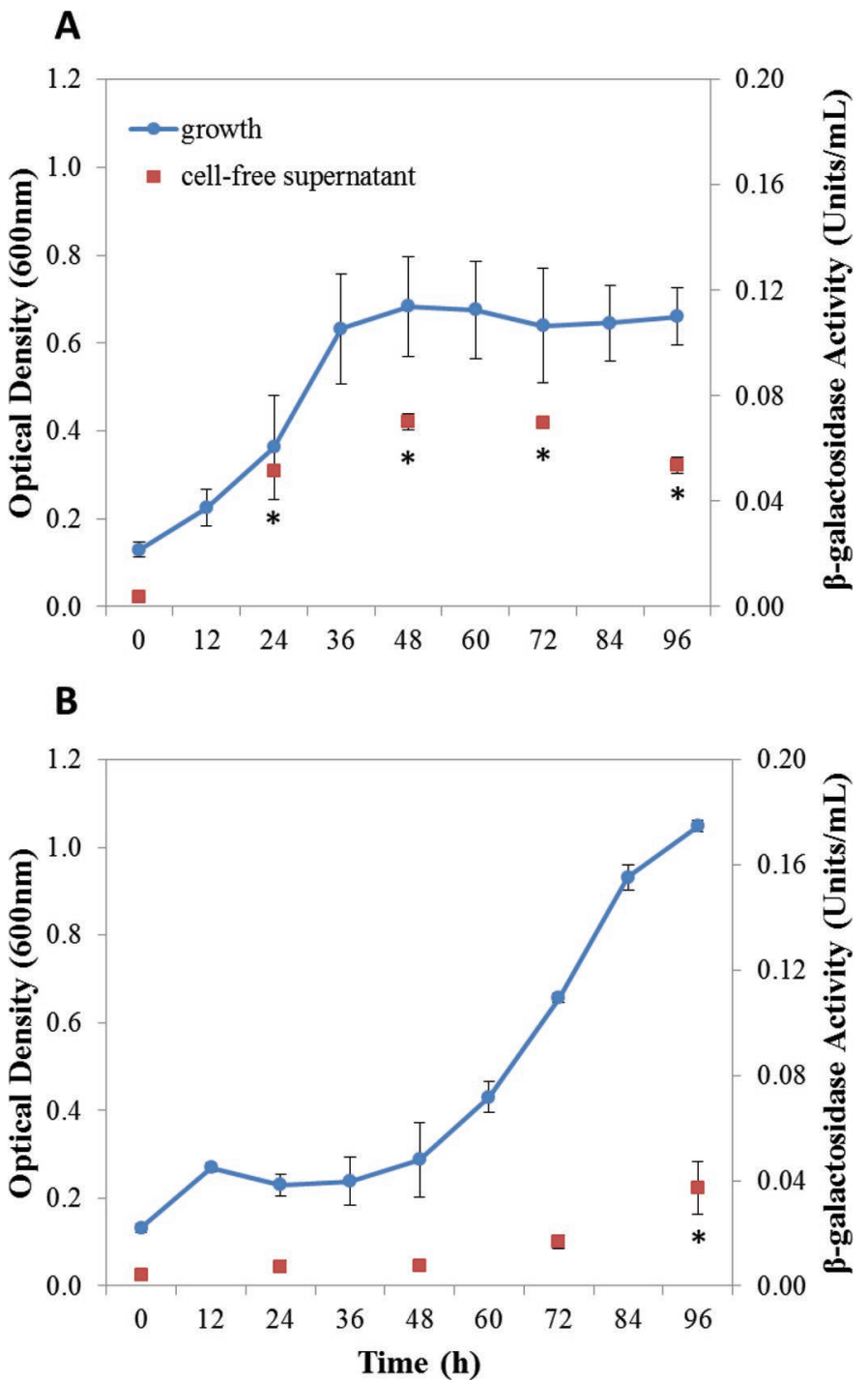

Figure 3. Extracellular $\beta$-galactosidase activity (units $/ \mathrm{mL}$ ) in the cell-free supernatants and growth (change in optical density at 600 $\mathrm{nm}$ ) of (A) Lactobacillus acidophilus NCFM and (B) L. acidophilus La-5 during growth in semidefined de Man, Rogosa and Sharpe broth supplemented with $1 \%$ lacto- $N$-neotetraose. Data are reported as the mean \pm standard error of 3 independent replicates. Values marked with an asterisk are significantly different $(P<0.05)$. Color version available online.

tested bifidobacteria and lactobacilli strains. Two probiotic strains, BI_M63 and LA_NCFM, were found to partially consume 2 '-FL and $\mathrm{LNnT}$, respectively. The accumulation of HMO constituent monomers (Fuc and LNTri) suggests a possible cross-feeding capacity in the GIT via liberated oligosaccharides. In addition, we characterized the function of the lacL $\beta$-galactosidase in the hydrolysis of LNnT by LA_NCFM. Overall, our results uncovered potential synbiotic combinations of pre- and probiotics. Future experiments should evalu- ate these synbiotic combinations in vivo to better understand HMO-microbe interactions in the GIT.

\section{ACKNOWLEDGMENTS}

We thank Todd R. Klaenhammer (North Carolina State University, Raleigh) for providing the tools to perform the $\beta$-galactosidase (lacL) knockout in Lactobacillus acidophilus NCFM. This work was partially supported by Abbott Nutrition (Columbus, OH). T. T. was supported with a fellowship from the Royal Thai Government.

\section{REFERENCES}

Andersen, J. M., R. Barrangou, M. Abou Hachem, S. Lahtinen, Y. J. Goh, B. Svensson, and T. R. Klaenhammer. 2011. Transcriptional and functional analysis of galactooligosaccharide uptake by lacS in Lactobacillus acidophilus. Proc. Natl. Acad. Sci. USA 108:1778517790. https://doi.org/10.1073/pnas.1114152108.

Asakuma, S., E. Hatakeyama, T. Urashima, E. Yoshida, T. Katayama, K. Yamamoto, H. Kumagai, H. Ashida, J. Hirose, and M. Kitaoka. 2011. Physiology of consumption of human milk oligosaccharides by infant gut-associated bifidobacteria. J. Biol. Chem. 286:3458334592. https://doi.org/10.1074/jbc.M111.248138.

Barrangou, R., E. Altermann, R. Hutkins, R. Cano, and T. R. Klaenhammer. 2003. Functional and comparative genomic analyses of an operon involved in fructooligosaccharide utilization by Lactobacillus acidophilus. Proc. Natl. Acad. Sci. USA 100:8957-8962. https://doi.org/10.1073/pnas.1332765100.

Bidart, G. N., J. Rodríguez-Díaz, and M. J. Yebra. 2015. The extracellular wall-bound $\beta$ - $N$-acetylglucosaminidase from Lactobacillus casei is involved in the metabolism of the human milk oligosaccharide lacto- $N$-triose. Appl. Environ. Microbiol. 82:570-577. https:// doi.org/10.1128/AEM.02888-15.

Bienenstock, J., R. H. Buck, H. Linke, P. Forsythe, A. M. Stanisz, and W. A. Kunze. 2013. Fucosylated but not sialylated milk oligosaccharides diminish colon motor contractions. PLoS One 8:e76236. https://doi.org/10.1371/journal.pone.0076236.

Bunesova, V., C. Lacroix, and C. Schwab. 2016. Fucosyllactose and Lfucose utilization of infant Bifidobacterium longum and Bifidobacterium kashiwanohense. BMC Microbiol. 16:248. https://doi.org/ 10.1186/s12866-016-0867-4.

Chow, J., M. R. Panasevich, D. Alexander, B. M. Vester Boler, M. C. Rossoni Serao, T. A. Faber, L. L. Bauer, and G. C. Fahey. 2014. Fecal metabolomics of healthy breast-fed versus formula-fed infants before and during in vitro batch culture fermentation. J. Proteome Res. 13:2534-2542. https://doi.org/10.1021/pr500011w.

Fernández, L., S. Langa, V. Martín, A. Maldonado, E. Jiménez, R Martín, and J. M. Rodríguez. 2013. The human milk microbiota: Origin and potential roles in health and disease. Pharmacol. Res. 69:1-10. https://doi.org/10.1016/j.phrs.2012.09.001.

Garrido, D., S. Ruiz-Moyano, N. Kirmiz, J. C. Davis, S. M. Totten, D. G. Lemay, J. A. Ugalde, J. B. German, C. B. Lebrilla, and D. A. Mills. 2016. A novel gene cluster allows preferential utilization of fucosylated milk oligosaccharides in Bifidobacterium longum ssp. longum SC596. Sci. Rep. 6:35045. https://doi.org/10.1038/ srep35045.

Garrido, D., S. Ruiz-Moyano, D. G. Lemay, D. A. Sela, J. B. German, and D. A. Mills. 2015. Comparative transcriptomics reveals key differences in the response to milk oligosaccharides of infant gut-associated bifidobacteria. Sci. Rep. 5:13517. https://doi.org/ $10.1038 /$ srep13517.

Ghose, T. K., and V. S. Bisaria. 1987. Measurement of hemicellulase activities: Part I Xylanases. Pure Appl. Chem. 59:1739-1752. https://doi.org/10.1351/pac198759121739. 
Good, M., C. P. Sodhi, Y. Yamaguchi, H. Jia, P. Lu, W. B. Fulton, L. Y. Martin, T. Prindle, D. F. Nino, Q. Zhou, C. Ma, J. A. Ozolek, R. H. Buck, K. C. Goehring, and D. J. Hackam. 2016. The human milk oligosaccharide 2 '-fucosyllactose attenuates the severity of experimental necrotising enterocolitis by enhancing mesenteric perfusion in the neonatal intestine. Br. J. Nutr. 116:1175-1187. https://doi.org/10.1017/S0007114516002944.

He, Y., S. Liu, S. Leone, and D. S. Newburg. 2014. Human colostrum oligosaccharides modulate major immunologic pathways of immature human intestine. Mucosal Immunol. 7:1326-1339. https://doi .org/10.1038/mi.2014.20.

Kocourek, J., M. Tichá, and J. Kostír. 1966. The use of diphenylamine-alanine-phosphoric acid reagent in the detection and differentiation of monosaccharides and their derivatives on paper chromatograms. J. Chromatogr. 24:117-124.

Kunz, C., S. Rudloff, W. Baier, N. Klein, and S. Strobel. 2000. Oligosaccharides in human milk: Structural, functional, and metabolic aspects. Annu. Rev. Nutr. 20:699-722. https://doi.org/10.1146/ annurev.nutr.20.1.699.

LoCascio, R. G., P. Desai, D. A. Sela, B. Weimer, and D. A. Mills. 2010. Broad conservation of milk utilization genes in Bifidobacterium longum ssp. infantis as revealed by comparative genomic hybridization. Appl. Environ. Microbiol. 76:7373-7381. https:// doi.org/10.1128/AEM.00675-10.

LoCascio, R. G., M. R. Niñonuevo, S. R. Kronewitter, S. L. Freeman, J. B. German, C. B. Lebrilla, and D. A. Mills. 2009. A versatile and scalable strategy for glycoprofiling bifidobacterial consumption of human milk oligosaccharides. Microb. Biotechnol. 2:333342. https://doi.org/10.1111/j.1751-7915.2008.00072.x.

Luchansky, J. B., M. C. Tennant, and T. R. Klaenhammer. 1991. Molecular cloning and deoxyribonucleic acid polymorphisms in Lactobacillus acidophilus and Lactobacillus gasseri. J. Dairy Sci. 74:3293-3302. https://doi.org/10.3168/jds.S0022-0302(91)78515 -9 .

Marcobal, A., M. Barboza, J. W. Froehlich, D. E. Block, J. B. German, C. B. Lebrilla, and D. A. Mills. 2010. Consumption of human milk oligosaccharides by gut-related microbes. J. Agric. Food Chem. 58:5334-5340. https://doi.org/10.1021/jf9044205.

Miwa, M., T. Horimoto, M. Kiyohara, T. Katayama, M. Kitaoka, H. Ashida, and K. Yamamoto. 2010. Cooperation of $\beta$-galactosidase and $\beta$ - $N$-acetylhexosaminidase from bifidobacteria in assimilation of human milk oligosaccharides with type 2 structure. Glycobiology 20:1402-1409. https://doi.org/10.1093/glycob/cwq101.

Newburg, D. S., G. M. Ruiz-Palacios, and A. L. Morrow. 2005. Human milk glycans protect infants against enteric pathogens. Annu. Rev. Nutr. 25:37-58. https://doi.org/10.1146/annurev.nutr.25.050304 .092553 .

Ninonuevo, M. R., Y. Park, H. Yin, J. Zhang, R. E. Ward, B. H. Clowers, J. B. German, S. L. Freeman, K. Killeen, R. Grimm, and C. B. Lebrilla. 2006. A strategy for annotating the human milk glycome. J. Agric. Food Chem. 54:7471-7480. https://doi.org/10 $.1021 /$ jf0615810.

Rodríguez-Díaz, J., V. Monedero, and M. J. Yebra. 2011. Utilization of natural fucosylated oligosaccharides by three novel alpha-L-fucosidases from a probiotic Lactobacillus casei strain. Appl. Environ. Microbiol. 77:703-705. https://doi.org/10.1128/AEM.01906-10.

Rodríguez-Díaz, J., A. Rubio-del-Campo, and M. J. Yebra. 2012. Lactobacillus casei ferments the $N$-acetylglucosamine moiety of fucosyl- $\alpha-1,3-N$-acetylglucosamine and excretes L-fucose. Appl. Environ. Microbiol. 78:4613-4619. https://doi.org/10.1128/AEM $.00474-12$.

Ruiz-Moyano, S., S. M. Totten, D. A. Garrido, J. T. Smilowitz, J. B. German, C. B. Lebrilla, and D. A. Mills. 2013. Variation in consumption of human milk oligosaccharides by infant gut-associated strains of Bifidobacterium breve. Appl. Environ. Microbiol. 79:6040-6049. https://doi.org/10.1128/AEM.01843-13.

Russell, W. M., and T. R. Klaenhammer. 2001. Efficient system for directed integration into the Lactobacillus acidophilus and Lactobacillus gasseri chromosomes via homologous recombination. Appl. Environ. Microbiol. 67:4361-4364.

Schwab, C., and M. Gänzle. 2011. Lactic acid bacteria fermentation of human milk oligosaccharide components, human milk oligosaccharides and galactooligosaccharides. FEMS Microbiol. Lett. 315:141148. https://doi.org/10.1111/j.1574-6968.2010.02185.x.

Sekirov, I., S. L. Russell, L. C. M. Antunes, and B. B. Finlay. 2010. Gut microbiota in health and disease. Physiol. Rev. 90:859-904. https://doi.org/10.1152/physrev.00045.2009.

Sela, D. A., J. Chapman, A. Adeuya, J. H. Kim, F. Chen, T. R. Whitehead, A. Lapidus, D. S. Rokhsar, C. B. Lebrilla, J. B. German, N. P. Price, P. M. Richardson, and D. A. Mills. 2008. The genome sequence of Bifidobacterium longum ssp. infantis reveals adaptations for milk utilization within the infant microbiome. Proc. Natl. Acad. Sci. USA 105:18964-18969. https://doi.org/10.1073/pnas .0809584105 .

Stahl, B., S. Thurl, J. Zeng, M. Karas, F. Hillenkamp, M. Steup, and G. Sawatzki. 1994. Oligosaccharides from human milk as revealed by matrix-assisted laser desorption/ionization mass spectrometry. Anal. Biochem. 223:218-226.

Thongaram, T., J. L. Hoeflinger, J. Chow, and M. J. Miller. 2017. Prebiotic galactooligosaccharide metabolism by probiotic lactobacilli and bifidobacteria. J. Agric. Food Chem. 65:4184-4192. https:// doi.org/10.1021/acs.jafc.7b00851.

Vázquez, E., A. Barranco, M. Ramírez, A. Gruart, J. M. DelgadoGarcía, E. Martínez-Lara, S. Blanco, M. J. Martín, E. Castanys, R. Buck, P. Prieto, and R. Rueda. 2015. Effects of a human milk oligosaccharide, 2'-fucosyllactose, on hippocampal long-term potentiation and learning capabilities in rodents. J. Nutr. Biochem. 26:455-465. https://doi.org/10.1016/j.jnutbio.2014.11.016.

Vester Boler, B. M., M. C. Rossoni Serao, T. A. Faber, L. L. Bauer, J. Chow, M. R. Murphy, and G. C. Fahey. 2013. In vitro fermentation characteristics of select nondigestible oligosaccharides by infant fecal inocula. J. Agric. Food Chem. 61:2109-2119. https://doi.org/ 10.1021/jf305056f.

Ward, R. E., M. Niñonuevo, D. A. Mills, C. B. Lebrilla, and J. B. German. 2006. In vitro fermentation of breast milk oligosaccharides by Bifidobacterium infantis and Lactobacillus gasseri. Appl. Environ. Microbiol. 72:4497-4499. https://doi.org/10.1128/AEM.02515-05.

Ward, R. E., M. Niñonuevo, D. A. Mills, C. B. Lebrilla, and J. B. German. 2007. In vitro fermentability of human milk oligosaccharides by several strains of bifidobacteria. Mol. Nutr. Food Res. 51:1398-1405. https://doi.org/10.1002/mnfr.200700150.

Yang, J., N. Vittori, W. Wang, Y.-C. Shi, J. L. Hoeflinger, M. J. Miller, and Y. Pan. 2017. Molecular weight distribution and fermentation of mechanically pre-treated konjac enzymatic hydrolysates. Carbohydr. Polym. 159:58-65. https://doi.org/10.1016/j .carbpol.2016.12.014.

Yu, Z.-T., C. Chen, and D. S. Newburg. 2013. Utilization of major fucosylated and sialylated human milk oligosaccharides by isolated human gut microbes. Glycobiology 23:1281-1292. https://doi.org/ $10.1093 /$ glycob/cwt065.

Zivkovic, A. M., J. B. German, C. B. Lebrilla, and D. A. Mills. 2011. Human milk glycobiome and its impact on the infant gastrointestinal microbiota. Proc. Natl. Acad. Sci. USA 108(Suppl. 1):46534658. https://doi.org/10.1073/pnas.1000083107. 